\title{
Current Status of and Perspectives on Cervical Cancer Screening in Korea
}

\author{
Sung-Chul Lim • Chong Woo Yoo ${ }^{1}$ \\ Department of Pathology, Chosun University \\ School of Medicine, Gwangju; ${ }^{1}$ Center for Uterine \\ Cancer and Department of Pathology, National \\ Cancer Center, Goyang, Korea \\ Received: January 3, 2019 \\ Revised: April 8, 2019 \\ Accepted: April 11, 2019 \\ Corresponding Author \\ Sung-Chul Lim, MD, PhD \\ Department of Pathology, Chosun University \\ Hospital, 365 Pilmun-daero, Dong-gu, Gwangju \\ 61453 , Korea \\ Tel: +82-62-230-6343 \\ Fax: +82-62-226-5860 \\ E-mail: sclim@chosun.ac.kr
}

Since the introduction of the Papanicolaou (Pap) smear system in 1943, cervicovaginal cytology has been used as a standard screening test for cervical cancer. The dissemination of this test contributed to reductions of the incidence and mortality of cervical cancer worldwide. In Korea, regular health check-ups for industrial workers and their family members were introduced in 1988 and were performed as part of the National Cancer Screening Program in 1999. As a result, the incidence of cervical cancer in Korea has been steadily decreasing. However, about 800 cases of cervical cancer-related deaths are reported each year due to false-negative test results. Hence, new screening methods have been proposed. Liquid-based cytology (LBC) was introduced in 1996 to overcome the limitations of conventional Pap smears. Since then, other LBC methods have been developed and utilized, including the human papilloma virus test-a method with higher sensitivity that requires fewer screenings. In this study, we review current issues and future perspectives related to cervical cancer screening in Korea.

Key Words: Uterus; Cervix uteri; Neoplasms; Screening; Papanicolaou test; Human papillomavirus DNA tests; Perspectives

\section{HISTORY OF CERVICAL CANCER SCREENING}

According to Globocan 2017 (International Agency for Research on Cancer [IARC]), the worldwide age-specific incidence rate of cervical cancer has been persistently decreasing, likely due to Papanicolaou (Pap) screening. ${ }^{1}$ Screening tests for cervical cancer have a long history, as cervical cancer, unlike other cancer types, is characterized by a long (7-20 years) precancerous stage prior to progression into an invasive cancer and easy detection is possible due to convenient existing diagnostic methods. Since the Pap smear system was first used by Dr. Papanicolaou in 1943 to examine cervicovaginal smears, it has been utilized as a screening test for cervical cancer. Most developed countries have comprehensively established and operated national cancer management programs. The Pap test (conventional smear [CS]) has several advantages as a screening tool for cervical cancer including low cost, simple procedures to obtain and manage specimens, and high specificity. The use of the Pap smear has contributed to reductions of the incidence and mortality of cervical cancer. ${ }^{2-4}$

In Korea, the Pap test (CS) was introduced in 1988 as a routine screening test for industrial workers and their family members, and has since played an important role in cervical cancer detection. In 1996, the Korean government established and promoted the 10-Year Plan to Conquer Cancer Project. In
1999, the National Cancer Screening Program included free health checkups for individuals to screen for three major types of cancer (gastric, breast, and cervical cancer).

In 2001, the National Cancer Center of Korea developed guidelines for the early detection of five major types of cancer (gastric, breast, cervical, hepatic, and colon cancer) under the supervision of the Ministry of Health and Welfare. The early screening guidelines for cervical cancer in the general population were also established. All sexually active females aged $\geq 20$ years were eligible for early screening of cervical cancer and were recommended to undergo cervicovaginal cytology. A nationwide campaign promoting early detection of cervical cancer was established in which females aged $\geq 30$ years were required to undergo cervicovaginal cytology bi-annually.

Therefore, cervicovaginal cytology was offered to the general Korean population enrolled in medical care (health insurance) programs in 1999. However, since 2002, low-income health insurance registrants (i.e., individuals registered in the health insurance program in the lowest $20 \%$ income bracket) have been eligible for bi-annual screening if they were 30 years of age or older. In addition, a legal basis to provide free screening for individuals among the lowest 30\% income bracket among health insurance registrants was established in 2003. Early screening of cervical cancer was made available to the general 
female population, as part of the bi-annual regular health checkup. The outcomes of early screening programs for cervical cancer were reported to The Bethesda System (2001). However, this did not include information regarding the duration of screening or age groups in which the test should be performed. ${ }^{5,6}$

The incidence of cervical cancer in the Korean population has been steadily decreasing, from 4,443 cases in 1999 (18.9 per 100,000 females, the third most common cancer affecting women) to 3,582 cases in 2015 (14.1 per 100,000 females, the seventh most common cancer affecting women). ${ }^{7}$ The Quality Improvement Committee of the Korean Society for Cytopathology (KSC) conducted nationwide quality control surveys in cytopathology in 1998, 2001, and 2016. Comparisons of the number of cervicovaginal cytology exams performed at medical centers (including university hospitals) and commercial laboratories participating in the survey showed that the majority of tests were performed in commercial laboratories $(66.4 \%$ in $1998,68.6 \%$ in 2001 , and $74.9 \%$ in 2016). The rate of cancer diagnosis from cervicovaginal cytology has remarkably decreased, from $0.1 \%-0.96 \%$ at university hospitals and $0.07 \%-0.09 \%$ at commercial laboratories in 1998 to $0.28 \%$ at university hospitals and $0.033 \%$ at commercial laboratories in $2016 .^{8,9}$ However, as about 800 cases of cervical cancer-related deaths are reported each year, concerns regarding limitations of current screening tests have been raised. ${ }^{7}$

Despite the numerous advantages of CS, this technique requires supplementation due to the high false-negative rate (20\%) caused by errors occurring during specimen collection, preservation, slide generation, analysis, and readout; low sensitivity; highly subjective results, and low reproducibility; and the level of expertise of the screener or pathologist. Hence, an improved screening tool is required. ${ }^{10,11}$

In Korea, about $60 \%$ of false-negative cases based on CS arise from specimen collection. To overcome these limitations, a liquid-based cytology (LBC) method involving fluid-based collection and processing was developed. Since the initial introduction of the ThinPrep Pap test (Cytyc Corp., Boxborough, MA, USA) in 1996, various LBC methods have been developed to improve the Pap test. In Korea, LBC-based cervicovaginal cytology has been covered by health insurance since 2006 .

LBC is thought to have the following advantages: low levels of drying artifacts, as each specimen is fixed immediately after collection; superior morphology; reduction in the number of cases with background obscuring caused by blood or inflammatory cells; quick and easy screening; better sampling due to the dispersion of cells; and the potential for multiple testing from a single sample. Previous clinical trials have demonstrated the effectiveness of LBC in increasing the rate of squamous intraepithelial lesion (SIL) detection, especially low-grade SIL (LSIL), and improving specimen adequacy. ${ }^{12,13}$

Several studies have compared the clinical utility of LBC-based cervicovaginal examination and outcomes using the CS method. The LBC method not only improved the diagnostic rate of atypical squamous cells of undetermined significance (ASCUS) and SIL, but also reduced the proportion of unsatisfactory samples, compared with the CS method. ${ }^{14-16}$

According to the results of the 2016 KSC survey, the most commonly used LBC Pap tests in Korea were as follows: ThinPrep (39.7\%), Cell Prep (26.3\%), SurePath (23.7\%), and other $(<5 \%)^{9}{ }^{9}$

A nationwide study conducted in Korea demonstrated that the number of LBCs performed during cervicovaginal cytology has steadily increased since the introduction of LBC, from $7.6 \%$ $(2004)^{4}$ to $20.5 \%(2007)^{4}$ to $25.3 \%$ (2015). ${ }^{9}$ According to the 2016 KSC survey, the CS method was more commonly used in commercial laboratories (CS, 79.4\%; LBC, 20.6\%) and general hospitals (CS, 80.9\%; LBC, 19.1\%), while the LBC method was more often used in university hospitals (CS, $44.1 \%$; LBC, 55.9\%).

Unsatisfactory samples in cervicovaginal cytology are usually reexamined within a period of $2-4$ months, since sampling adequacy allows for appropriate patient follow-up. ${ }^{17}$ Appropriate comparisons could not be made, since there is a limited number of Korean studies assessing unsatisfactory samples. However, according to the 2016 KSC survey, unsatisfactory samples accounted for $0.6 \%$ of all cervicovaginal cytology cases. ${ }^{9}$ As LBC can reduce the proportion of unsatisfactory samples, the proportion of unsatisfactory samples will likely decrease from $0.6 \%$ in the future. ${ }^{18}$

Moreover, diagnostic accuracy assessed by comparing cytologic cases with matching histological specimens is crucial for evaluations of false positives/negatives. The diagnostic accuracy values reported in the 2016 KSC survey were as follows: for university hospitals, category A, 9.1\%; category B, 4.0\%; category C, $0.6 \%$; and category $\mathrm{O}, 86.3 \%$; and for general hospitals, category A, $17.4 \%$; category B, $10.2 \%$; category C, $1.9 \%$; and category $\mathrm{O}, 69.5 \%$. Accuracy data from commercial laboratories were not available. ${ }^{9}$

Since Pap tests alone cannot produce accurate outcomes for cervical cancer screening, an adjunctive, combined test is required. ${ }^{19}$ The combination of Pap test and colposcopy is reported to increase sensitivity, but the limitations of colposcopy (cost, time, and expert training) make it impractical for use as a screening method. ${ }^{20,21}$ Similar to colposcopy, cervicography is another 
potential adjunctive test to complement Pap tests, allowing for interpretations of ectocervical photographic images. Evaluations by cervicography are based on the principles of colposcopy. ${ }^{21,22}$

Higher diagnostic accuracy can be achieved by utilizing the Pap test and cervicography as a combined screening method: 90\%-100\% for infiltrating cancers and 90\%-95\% for cervical intraepithelial neoplasia (CIN). However, its medical cost, despite being less than a combined method using the Pap test and human papillomavirus (HPV) test, is still a significant limiting factor for it to be used as a general screening method. ${ }^{23}$

HPV plays an important role in the development of cervical cancer and is found in $90 \%-100 \%$ of high-grade SIL (HSIL) or invasive carcinomas. Therefore, including HPV tests in cervical cancer screening is useful for complementing traditional cytologic examinations. ${ }^{24}$ More specifically, for the epidemiological management of cervical cancer, screening of patients diagnosed with ASCUS is a clinically important issue. Repeat cervicovaginal smear tests, colposcopy, and HPV DNA tests are recommended methods to select high-risk patients diagnosed with ASCUS, and the utility of HPV DNA test is steadily emphasized.

There are two major HPV examination methods: the first involves amplifying HPV DNA by polymerase chain reaction (PCR), while the second involves direct confirmation of HPV DNA through DNA hybridization. In the 1994 Interim Guidelines, HPV tests were suggested as an adjunctive method for the management of ASCUS and LSIL. ${ }^{25}$ However, the reliability of HPV tests has been questioned due to the diverse sensitivity of this examination method. The sensitivity and reliability of the Hybrid Capture II system (Digene Corporation, Qiagen N.V., Venlo, Netherlands) have been approved by the Food and Drug Administration (FDA), and it has been widely used to test for HPV. An HPV DNA microchip test that allows for the identification of HPV genotypes from a single examination is also being utilized.

Although the majority of ASCUS or LSIL are naturally eliminated, a small portion persist or develop into more severe CIN. Furthermore, among patients initially diagnosed with ASCUS or LSIL by the Pap test, 5\%-15\% are found to have HSIL through biopsy, ${ }^{26}$ suggesting that it is important to check for HSIL in patients diagnosed with ASCUS or LSIL by the Pap test.

According to a previous study, ${ }^{27} \mathrm{HPV}$ tests had a greater sensitivity for detecting HSIL from ASCUS than Pap tests ( 0.83 vs 0.66), but combination of the HPV test and Pap test has increased sensitivity (0.92). The Pap test (1.00) has higher sensitivity for detecting HSIL from LSIL compared with the HPV test (0.93), while a combination of HPV and Pap tests also showed a sensi- tivity of 1.00. Moreover, the ASCUS-LSIL Triage Study (ALTS) determined that HPV tests are useful for the management of ASCUS patients but not useful for the management of LSIL patients; thus, LSIL patients should undergo immediate colposcopy or Pap tests every 6 months. ${ }^{28,29}$

A joint study performed by a Korean group and IARC selected a random region in the city of Busan to evaluate the prevalence of HPV infections via cervicovaginal cytology (PCR-enzyme immunoassay method). This study showed that $10.3 \%$ of participants were positive for HPV DNA, while $60 \%$ had high-risk HPV (HR-HPV). The HR-HPV types observed among infected individuals in order of frequency were HPV 16, 33, 58, 66, 18, 31 , and other, while the low-risk HPV (LR-HPV) types observed in order of frequency were HPV 70, 81, and other. ${ }^{30}$

Unlike studies of the general population, one study that selected a specific cohort of patients from the Department of Obstetrics and Gynecology at a university hospital in Korea and evaluated the prevalence of HPV infections via cervicovaginal cytology (HPV DNA microchip test). Interestingly, $48.8 \%$ of the participants were positive for HPV DNA, while $86.9 \%$ were positive for HR-HPV. The HR-HPV types detected among infected individuals in order of frequency were HPV 16, 58, 18, 52, 53, 31, and other, while the LR-HPV types detected in order of frequency were HPV 70, 6, 11, 40, and $42 .{ }^{31}$ Therefore, there were differences between the general population cohort and a cohort of patients from the Department of Obstetrics and Gynecology in HR-HPV and LR-HPV types.

\section{CURRENT STATUS OF CERVICAL CANCER SCREENING}

Cervical cancer screening tests using cervicovaginal cytology were not widely used in the early stages of screening programs. According to a 1998 survey performed by the Ministry of Health and Welfare in Korea, only $33.88 \%$ of women ( $\geq 20$ years old) underwent screening tests, likely due to the lack of promotion and follow-up for lower-income brackets. ${ }^{7}$ Since then, promotion among the general population and the provision of free screening tests for cervical cancer have steadily increased the rate of eligible women undergoing screening.

Cervical cancer screening is covered by international guidelines provided by the World Health Organization and the European Commission. A few European countries, including France, have established new screening programs for 2014-2019, based on pilot experiments performed in different regions of participating countries. ${ }^{32}$ Such programs will initially be based on the 
Pap test, but with the objective of progressing toward direct screening for HPV infection.

A German study that assessed costs and clinical effectiveness showed that HR-HPV (HPV 16/18) tests performed in conjunction with p16/Ki-67 dual-stained cytology can improve the detection rate of cervical cancer compared to the Pap test alone, with lower total annual cost. ${ }^{33}$ In addition, an Australian study demonstrated that HPV tests performed every 5 years are more effective for cervical cancer screening and cost-savings than Pap tests performed every 2 years. This Australian National Cervical Screening Program switched to primary HPV screening with partial genotyping as of $2017 . .^{34}$

Even in the United States, where the use of combined Pap plus HPV test is recommended, additional studies have been performed to determine which screening method can best improve clinical and economic outcomes, since the approval of the HPV test in 2014 as a primary cervical cancer screening tool for women aged $\geq 25$ years. The outcomes of related studies have reported that primary HR-HPV screening performed every 5 years is the most efficient alternative screening tool to the Pap test. ${ }^{35}$

Most updated guidelines for cervical cancer screening in Korea recommend that all asymptomatic women aged over 20 years should start cervical cancer screening by Pap smear (CS or LBC) and continue every 3 years until they reach the age of 74 if three consecutive cytologic examinations have been negative within the previous 10 years. ${ }^{36}$

These fundamental differences in strategies of cervical cancer screening between Korea and other countries (US, Australia, and European countries) are likely due to insufficient screening or poor accessibility to medical services in other countries. As a consequence, Pap tests are underused in underserved populations, and there are inequalities in access to the Pap test. The cost of HPV testing is lower than the costs associated with the establishment of cytological screening systems. In addition, noncompliant populations and individuals may perform self-sampling as they cannot meet medical staff frequently. Hence, HPV tests are recommended screening methods in low-access countries. ${ }^{37}$

Although HPV testing is increasingly used for primary screening in some countries, its efficacy and cost-effectiveness vary in different clinical and social-economical settings. The Pap test is extremely useful in countries with well-developed screening systems or in populous countries like Korea. In Japan, women between 20 and 69 years of age undergo cytologic screening every 2 years. ${ }^{38}$ In Hong Kong, women more than 25 years of age undergo cytologic screening at one- to three-year intervals, and cervical cytology remains the most effective screening tool for population-based cervical cancer screening. ${ }^{39}$ Taiwan also provides cytologic screening for women more than 30 years of age at 3 -year intervals. ${ }^{40}$

Due to limited financial support from the national cancer screening program and the low associated medical costs when performed in obstetrics and gynecology clinics, the Pap test is still often utilized in Korea. The Pap test is considered the core of cervical cancer screening, due to the following factors: nationwide quality assurance management of Pap tests via the Korean Society for Cytopathology, available training for qualified cytology screening personnel, low accuracy of the HPV test, low reproducibility between HPV tests, and relatively high cost associated with the HPV test. Furthermore, even in HPV-negative cases, lesions equal to or more severe than mild dysplasia are observed under cytological and histopathological examinations in 17.5\% of cases. ${ }^{31}$ Finally, despite the high sensitivity of the HPV test, it has the crucial disadvantage of low specificity and its standalone outcome is not useful in most cases, which may cause unnecessary anxiety for patients. Therefore, in the 2015 guidelines for cervical cancer screening provided by the Ministry of Health and Welfare in Korea, the HPV test is not considered an adequate standalone screening test. ${ }^{36}$

\section{FUTURE PERSPECTIVES ON CERVICAL CANCER SCREENING IN KOREA}

Korea has socioeconomic, geographical, and ethnic differences from other countries (Australia, North American, and European countries), where the HPV test is used as a primary cervical cancer screening tool. Therefore, actual conditions in Korea must be considered prior to the establishment of new cervical cancer screening methods.

There are no systematic studies regarding the reproducibility and accuracy of HPV tests used in Korea. The uncontrolled performance of HPV tests in Korea therefore might result in increased medical costs and patient anxiety. To determine whether HPV tests should be used as primary cervical cancer screening methods in Korea, a nationwide quality assurance management protocol for HPV testing should be established, and study outcomes regarding the accuracy and relative sensitivity of HPV tests should be made available to the public. Consequently, institutions performing HPV tests should choose a validated, acceptable screening method, and quality control of screening methods must be ensured prior to the utilization of HPV tests. In addition, the health insurance costs of HPV tests are extremely high (53,480 won) compared to the Pap test (7,750 won), which is an 
important obstacle to the establishment of a nationwide cervical cancer screening program.

A recently published meta-analysis confirmed the diagnostic accuracy of cytology as a primary screening test, which has higher sensitivity and specificity for the detection of SIL and squamous cell carcinoma. ${ }^{41}$ Therefore, cervicovaginal cytology is one of the most useful, sensitive, and confirmative primary cervical cancer screening tests available.

The revised guidelines for cervical cancer screening in Korea (2015) can be summarized as follows: "The committee recommends screening for cervical cancer in women older than 20 years of age with cytology (Pap test or LBC) every 3 years (recommendation A). The combination test (cytology with HPV test) is recommended as an option in consideration of individual risks or preferences (recommendation C). The current evidence for primary HPV screening is insufficient to assess the benefits and harm of cervical cancer screening (recommendation I). Cervical cancer screening can be terminated at 74 years old if it has been confirmed that the patient has more than 3 consecutive negative cytology results within 10 years. ${ }^{~}{ }^{36}$ As described above, HPV tests in Korea are considered useful co-tests when performed alongside the Pap test, rather than as a primary cervical cancer screening method.

Aside from the cervical cancer screening methods mentioned above, HPV vaccines, which were approved by the FDA in 2006 and have been included as a part of a cost-free vaccine program for 12-year-old girls in Korea as of 2016, will likely prevent future cases of cervical cancer. However, current commercial HPV vaccines showed limited effects. Gardasil (Merck, NJ, USA) showed limited effects against specific viruses including HPV 6,11,16, and 18. Gardasil 9 (Merck) showed limited effects against HPV $6,11,16,18,31,33,45,52$, and 58, while Cervarix (GSK, London, UK) showed limited effects against HPV 16 and 18. HPV types 16 and 18 account for between $55 \%$ and $70 \%$ of cancers and mainly cause cervical cancers. At least $30 \%$ of cancers contain other HR-HPV types and would not be prevented by current vaccines. ${ }^{42}$

Thus, even if HPV vaccines become publicly available, both non-vaccinated women and vaccinated women will need to undergo regular cervical screening using the best, most sensitive and specific available screening tools available in the current era. Moreover, current commercial HPV vaccines have no effect against the prevalent HR-HPV 53/66 and LR-HPV 70 strains in cohort studies, ${ }^{30,31}$ thus demonstrating limitations to the application of commercial HPV vaccines in Korea. Thus, a more effective HPV vaccine must be developed in Korea, based on epide- miological studies of larger Korean population and regional samples.

\section{ORCID}

Sung-Chul Lim: https://orcid.org/0000-0001-6179-691X

Chong Woo Yoo: https://orcid.org/0000-0002-5221-4516

\section{Author Contributions}

Conceptualization: SCL.

Data curation: SCL, CWY.

Formal analysis: SCL, CWY.

Investigation: SCL, CWY.

Methodology: SCL, CWY.

Project administration: SCL, CWY.

Writing—original draft: SCL.

Writing—review \& editing: SCL, CWY.

\section{Conflicts of Interest}

The authors declare that they have no potential conflicts of interest.

\section{Acknowledgments}

This study was supported by research fund from Chosun University Hospital, 2018.

\section{REFERENCES}

1. The Union for International Cancer Control (UICC). 2017 GLOBOCAN annual report [Internet]. Geneva: Union for International Cancer Control, 2017 [cited 2019 Jan 2]. Available from: http:/ / www.uicc.org/2017-annual report.

2. Devesa SS, Young JL Jr, Brinton LA, Fraumeni JF Jr. Recent trends in cervix uteri cancer. Cancer 1989; 64: 2184-90.

3. Pretorius R, Semrad N, Watring W, Fotheringham N. Presentation of cervical cancer. Gynecol Oncol 1991; 42: 48-53.

4. Lee HK, Kim SN, Khang SK, Kang CS, Yoon HK. Quality control program and its results of Korean Society for Cytopathologists. Korean J Cytopathol 2008; 19: 65-71.

5. Oh DK. National cancer control programs: the review of the National Cancer Screening Program. J Korean Med Assoc 2003; 46: 668-9.

6. Shin HR, Ahn YO, Bae JM, et al. Cancer incidence in Korea. Cancer Res Treat 2002; 34: 405-8.

7. National Cancer Control Institute. Annual report of cancer statistics in Korea in 2015 [Internet]. Goyang: National Cancer Center, 2017 [cited 2019 Jan 2]. Available from: http://ncc.re.kr/main.ncc?uri= 
manage02_1.

8. Park MH. Cervical cancer screening in Korea. Korean J Cytopathol 2003; 14: 43-52.

9. Oh EJ, Jung CK, Kim DH, et al. Current cytology practices in Korea: a nationwide survey by the Korean Society for Cytopathology. J Pathol Transl Med 2017; 51: 579-87.

10. Dodd LG, Sneige N, Villarreal Y, et al. Quality-assurance study of simultaneously sampled, non-correlating cervical cytology and biopsies. Diagn Cytopathol 1993; 9: 138-44.

11. Bishop JW, Bigner SH, Colgan TJ, et al. Multicenter masked evaluation of AutoCyte PREP thin layers with matched conventional smears. Including initial biopsy results. Acta Cytol 1998; 42: 189-97.

12. Hutchinson ML, Zahniser DJ, Sherman ME, et al. Utility of liquidbased cytology for cervical carcinoma screening: results of a population-based study conducted in a region of Costa Rica with a high incidence of cervical carcinoma. Cancer 1999; 87: 48-55.

13. Linder J. Recent advances in thin-layer cytology. Diagn Cytopathol 1998; 18: 24-32.

14. Lee KR, Ashfaq R, Birdsong GG, Corkill ME, McIntosh KM, Inhorn SL. Comparison of conventional Papanicolaou smears and a fluidbased, thin-layer system for cervical cancer screening. Obstet Gynecol 1997; 90: 278-84.

15. Papillo JL, Zarka MA, St John TL. Evaluation of the ThinPrep Pap test in clinical practice: a seven-month, 16,314-case experience in northern Vermont. Acta Cytol 1998; 42: 203-8.

16. Austin RM, Ramzy I. Increased detection of epithelial cell abnormalities by liquid-based gynecologic cytology preparations: a review of accumulated data. Acta Cytol 1998; 42: 178-84.

17. Wright TC Jr, Cox JT, Massad LS, Twiggs LB, Wilkinson EJ, Conference AS-SC. 2001 Consensus Guidelines for the management of women with cervical cytological abnormalities. JAMA 2002; 287: 2120-9.

18. Jeong H, Hong SR, Chae SW, et al. Comparison of unsatisfactory samples from conventional smear versus liquid-based cytology in uterine cervical cancer screening test. J Pathol Transl Med 2017; 51: 314-9.

19. Reid R, Greenberg MD, Lorincz A, et al. Should cervical cytologic testing be augmented by cervicography or human papillomavirus deoxyribonucleic acid detection? Am J Obstet Gynecol 1991; 164(6 Pt 1): 1461-9.

20. Stafl A. Cervicography: a new method for cervical cancer detection. Am J Obstet Gynecol 1981; 139: 815-25.

21. Hocutt JE Jr, Clark RR, Pfenninger JL, Queripel P. Papanicolaou testing and colposcopic screening. J Fam Pract 1992; 34: 38-40.

22. Ferris DG, Willner WA, Ho JJ. Colpophotography systems: a review. J Fam Pract 1991; 33: 633-9.
23. Kim SJ, Park CK, Lee HP, et al. Multiinstitutional study for the development of cervical cancer screening model with new cervicography, Pap smear and HPV-DNA test. Korean J Obstet Gynecol 2001; 44: 911-37.

24. Park JS. Biology and oncogenesis of human papillomavirus and its clinical application. Korean J Cytopathol 2004; 15: 1-10.

25. Kurman RJ, Henson DE, Herbst AL, Noller KL, Schiffman MH. Interim guidelines for management of abnormal cervical cytology. The 1992 National Cancer Institute Workshop. JAMA 1994; 271: $1866-9$.

26. Nyirjesy I, Billingsley FS, Forman MR. Evaluation of atypical and low-grade cervical cytology in private practice. Obstet Gynecol 1998; 92 (4 Pt 1): 601-7.

27. Bergeron C, Jeannel D, Poveda J, Cassonnet P, Orth G. Human papillomavirus testing in women with mild cytologic atypia. Obstet Gynecol 2000; 95(6 Pt 1): 821-7.

28. Human papillomavirus testing for triage of women with cytologic evidence of low-grade squamous intraepithelial lesions: baseline data from a randomized trial. The Atypical Squamous Cells of Undetermined Significance/Low-Grade Squamous Intraepithelial Lesions Triage Study (ALTS) Group. J Natl Cancer Inst 2000; 92 : 397-402.

29. Schiffman M, Solomon D. Findings to date from the ASCUS-LSIL Triage Study (ALTS). Arch Pathol Lab Med 2003; 127: 946-9.

30. Hong SH, Lee DH, Shin HR. Prevalence of human papillomavirus infection in women in South Korea: incidence of positive HPV DNA and anti-VLPs in residents of Busan City. Korean J Cytopathol 2004; 15: 17-27.

31. Jeong JH, Cho HY, Kim NR, Chung DH, Park S, Ha SY. The analysis and clinical usefulness of HPV DNA chip test in the uterine cervix. Korean J Pathol 2010; 44: 77-82.

32. Institut National du Cancer. 2014-2019 Cancer plan, cure and prevent cancer: give to all and anywhere in France the same opportunities. Boulogne-Billancourt: Institut National du Cancer, 2014; 77-9.

33. Petry KU, Barth C, Wasem J, Neumann A. A model to evaluate the costs and clinical effectiveness of human papilloma virus screening compared with annual papanicolaou cytology in Germany. Eur J Obstet Gynecol Reprod Biol 2017; 212: 132-9.

34. Lew JB, Simms KT, Smith MA, et al. Primary HPV testing versus cytology-based cervical screening in women in Australia vaccinated for HPV and unvaccinated: effectiveness and economic assessment for the National Cervical Screening Program. Lancet Public Health 2017; 2: e96-107.

35. Kim JJ, Burger EA, Regan C, Sy S. Screening for cervical cancer in primary care: a decision analysis for the U.S. Preventive Services Task Force [Internet]. Rockville: Agency for Healthcare Research 
and Quality, 2018 [cited 2019 Jan 2]. Available from http://www. ncbi.nlm.nih.gov/books/NBK525069/.

36. Min KJ, Lee YJ, Suh M, et al. The Korean guideline for cervical cancer screening. J Gynecol Oncol 2015; 26: 232-9.

37. Haguenoer K, Giraudeau B, Gaudy-Graffin C, et al. Accuracy of dry vaginal self-sampling for detecting high-risk human papillomavirus infection in cervical cancer screening: a cross-sectional study. Gynecol Oncol 2014; 134: 302-8.

38. HPV Information Center. Japan: human papillomavirus and related cancers, fact sheet 2018. Barcelona: ICO/IARC Information Centre on HPV and Cancer, 2018.

39. The Hong Kong College of Obstetricians and Gynaecologists. Guidelines for cervical cancer prevention and screening [Internet].
Hong Kong: The Hong Kong College of Obstetricians and Gynaecologists, 2016 [cited 2019 Jan 2]. Available from: http:/ /www.hkcog.org.hk/hkcog/Download/Cervical_Cancer_Prevention_and_ Screening_r evised_November_2016.pdf.

40. Chen MJ, Wu CY, Chen R, Wang YW. HPV vaccination and cervical cancer screening in Taiwan. J Global Oncol 2018; 4 Suppl 2: $235 S$.

41. Pyo JS, Kang G, Yoon HK, Kim HJ. Diagnostic test accuracy review of cytology for squamous intraepithelial lesion and squamous cell carcinoma of uterine cervix. J Korean Med Sci 2019; 34: e16.

42. McGoogan E. Cervical cancer prevention for the future: the complimentary roles of cytology screening and HPV vaccination. Korean J Cytopathol 2007; 18: 1-12. 